\title{
Preventing acute infection in total hip prostheses implanted after external fixation of the femur: is there a need for a staged procedure?
}

\author{
A. Massè • A. Aprato • E. Guzzi Susini
}

Received: 6 February 2007 / Accepted: 22 October 2007 / Published online: 17 March 2008

\begin{abstract}
We report two cases of acute infection of an uncemented femoral component in a hip prosthesis implanted after external fixation of a femoral fracture. In both cases, the surgical access did not cross over the pin scars. When the prosthesis was implanted the stem crossed one or more pin tracts. The preoperative clinical examination, laboratory tests and bone scintigraphy with marked granulocytosis did not show signs of local infection in either case. We suggest that every patient destined to receive a prosthesis after external fixation should be treated with a staged procedure, the first step being excision of the soft tissues around the pin tracts and curettage or drilling of the bony holes, followed by prosthesis implant.
\end{abstract}

Keywords Infection · External fixation · Prosthesis

\section{Introduction}

According to the criteria of "damage control orthopaedics" (DCO), external fixation is a frequently adopted temporary treatment used in patients with multitrauma. However, due to the high complication rate related to the pin tracts, it is seldom used as a definitive treatment for femoral fractures. When not used as a definitive treatment, external fixation is commonly converted to intramedullary nailing. However, temporary external fixation increases the risk of infection in late definitive treatment, in $1.7 \%-10 \%$ of cases [1-3]. The infection risk, determined by a high grade of bacterial colonization of the pin tracts [4-7], is directly related to the duration of the external fixation. This observation leads to the hypothesis that the implant of a hip prosthesis after external fixation may pose a high risk of infection. We report two cases of acute infection of the uncemented femoral component of a hip prosthesis implanted after previous synthesis of a femoral fracture by external fixation.

\section{Case reports}

\section{Case 1}

\footnotetext{
A. Massè · A. Aprato (凶)

First Clinic of Orthopaedics and Traumatology

University of Turin

Turin, Italy

e-mail: alessandro.aprato@virgilio.it

E. Guzzi Susini

Traumatology Department

Orthopaedic and Traumatological Hospital of Turin

Turin, Italy
}

A 35-year-old woman had a left acetabular and femoral fracture, pneumothorax and spleen rupture, with an injury severity score of 17 , as the result of a car accident in November 1999. Urgent laparotomy and femoral stabilization with an external fixator (Hoffman II, Stryker Howmedica) were performed elsewhere (Fig. 1a). Two weeks later, the external fixator was converted into an intramedullary nail and acetabular synthesis was attempt- 

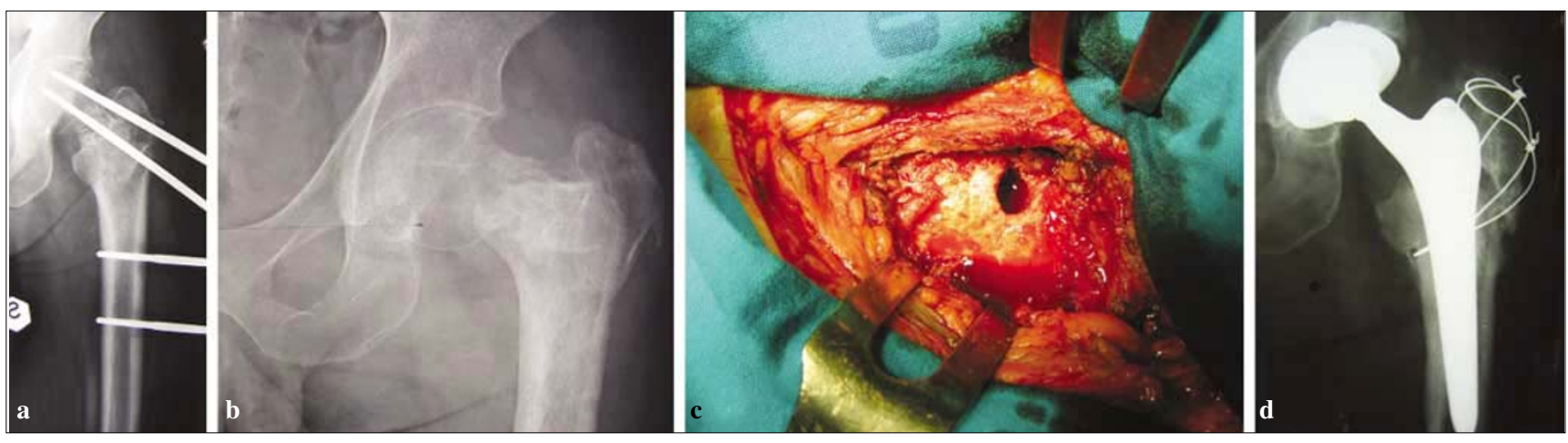

Fig. 1a-d Case 1. a Femoral stabilization with external monolateral fixator after urgent laparotomy. b One year after the accident, radiograph shows severe hip osteoarthritis. c Intraoperative view of screw's hole during hip prosthesis implant. d Postoperative radiograph after surgical debridment

ed. Twelve months after the trauma, the nail was removed and a monolateral external fixator (Orthofix) was applied due to the presence of pseudoarthrosis. The fracture healed in 5 months and the fixator was removed.

The patient came to our attention one year later due to severe left osteoarthritis (Fig. 1b). There was no sign of local inflammation at the pin tract sites. Laboratory tests performed at admission showed normal levels of inflammatory markers and normal neutrophil counts. Tc99 focal scintigraphy was performed to exclude osteitis, and gave no remarkable results except for accumulation of labeled white blood cells. An uncemented hip prosthesis was implanted using a posterolateral approach to avoid the pin tract scars (Fig. 1c). Routine antibiotic prophylaxis was administered. The post-operative course was unremarkable and the patient was discharged seven days after the procedure.

Two weeks later, the patient returned to our hospital complaining of fever and swelling of the surgical wound. Laboratory tests showed an erythrocyte sedimentation rate (ESR) of $15 \mathrm{~mm} / \mathrm{h}$. Fibrinogen was 401 $\mathrm{mg} / \mathrm{dl}$ (normal range, 150-450) and C-reactive protein was $3.2 \mathrm{mg} / \mathrm{l}$ (normal values, $<5$ ). Neutrophil count was of $3.49 \times 10^{9} / 1$ (normal range, $1.5-6.0 \times 10^{9} / 1$ ) and the percentage of neutrophils $(53.6 \%)$ was elevated. Tc99 scintigraphy showed a focal captation near the greater trochanter around one of the pin tracts. Surgical debridement of the wound with en bloc excision of the pin tract and the surrounding bone was performed (Fig. 1d). Histological examination of the bone specimens revealed the presence of a small ring enclosed in fibrous tissue. Cultures grew methicillin-resistant Staphylococcus aureus. Systemic antibiotic therapy was administered, with vancomycin ( $1 \mathrm{~g}$ twice daily) and rifampin (600 mg once daily), for three months. At the one-year follow-up, the patient was asymptomatic with normal laboratory test results.
Case 2

A 42-year-old man had a bilateral femoral fractures, liver rupture, right tibial pilon fracture, head injury and left hemothorax as the result of a motorcycle accident. The treatment adopted was external fixation for all the fractures, according to DCO criteria. The left femoral fracture was bifocal with midcervical involvement and was initially treated by implanting a monolateral external fixator (Orthofix) with proximal screws along the femoral neck. The severity of the patient's general health did not allow for conversion of this treatment.

All but one of the fractures healed within five months, with a non-union in the midcervical fracture. The patient underwent hip replacement four months after removal of the external fixator through a posterolateral approach. The postoperative course was unevenful and the patient was discharged after 6 days.

Twenty days later the patient returned to our observation complaining of swelling and the appearance of a fistula along the surgical wound. Cultures grew methicillinresistant Staphylococcus aureus. A two-step revision procedure was performed and antibiotic therapy was administered for four months between implant removal and revision (600 mg teicoplanin twice daily and $1 \mathrm{~g}$ ceftriaxone twice daily). Clinical evaluation and laboratory tests performed at 18 months, showed no evidence of persisting infection and, at the time of writing, the patient is considered healed.

Other cases in our experience

Total hip prosthesis was used in two other cases of osteoarthritis on femurs that had previously been treated with external fixators. The laboratory tests were within the normal ranges in both cases. The first step carried out, 
two months before prosthesis implantation, was surgical cleansing of the bone pin-tracts and excision of the scars in both cases by drilling, using a larger bit than had previously been used. The bacteriological examination was positive for Staphylococcus epidermidis in one case and Staphylococcus aureus in the other. Therefore, a period of systemic antibiotic therapy was administred before hip replacement. The postoperative course was normal in normal cases.

\section{Discussion}

External fixation significantly increases he risk of infection of any subsequent surgical procedure. Pin-tract infection occurs in $2 \%-30 \%$ of cases [4-7]. Indeed, the overall percentage of bacterial colonization of the screw tips seems to be even higher. Mahan et al. [8] reported that $75 \%$ of examined screw tips cultured positive at removal, with a higher rate of gram-positive, than gram-negative bacteria. In a recent study performed in our institute [6], 56 screws were removed and $43 \%$ of the screw tips gave a positive result. Staphylococcus aureus was found in $48.7 \%$ of the cases. These observations raise the question as to the possible infection of internal devices implanted after external fixation.

In the presented cases, the preoperative clinical exam, laboratory tests and bone scan with marked granulocytosis showed no signs of local infection. Although clinicians consider ESR a sensitive laboratory indicator of pyogenic infection, it may be negative in up to $10 \%$ of patients with bone infection and is, therefore, not specific for infection [10]. CRP may not be elevated in up to $10 \%$ of patients with bone infection, even if it may be more specific than ESR [10]. Therefore, it can be assumed that, although these markers serve both as good screening aids and surveillance tests in the diagnosis and treatment of bone infections, a negative result does not necessarily exclude the presence of infection. Indeed, some authors in recent reviews concluded that the white blood cell count is not particularly useful alone for the diagnosis of a bone infection, but should rather be part of an infection-fever workup, as it may provide some general guidance as to treatment response [9, 10]. Although the most helpful examination is leukocyte scintigraphy, it has an $80 \%$ sensitivity and an $80 \%$ for the detection of chronic osteomyelitis in the peripheral skeleton [10].

Therefore, we conclude that a subclinical infection cannot be excluded with the adoption of these techniques. We suggest that every patient destined to receive a prosthesis after external fixation undergo a surgical excision of the pin tracts before implantation. To the best of our knowledge, there are no reports of hip prostheses implanted after external fixation of the femur. From our experience with these cases, it seems preferable to treat such patients with a staged procedure, the first step being excision of the soft tissues around the pin tracts and curettage and drilling of the bony holes.

\section{References}

1. Harwood PJ, Giannoudis PV, Probst C et al (2006) The risk of local infective complications after damage control procedures for femoral shaft fracture. J Orthop Trauma 20:181-189

2. Yokoyama K, Uchino M, Nakamura K et al (2005) Risk factors for deep infection in secondary intramedullary nailing after external fixation for open tibial fractures. Injury 37:554-560

3. Nowotarski PJ, Turen CH, Brumback RJ, Scarboro JM (2000) Conversion of external fixation to intramedullary nailing for fractures of the shaft of the femur in multiply injured patients. J Bone Joint Surg Am 82:781-788

4. Temple J, Santy J (2004) Pin site care for preventing infections associated with external bone fixators and pins. Cochrane Database Syst Rev 1:CD004551

5. Moroni A, Vannini F, Mosca M, Giannini S (2002) Pin loosening and infection in external fixation. J Orthop Trauma 16:189-195

6. Massè A, Bruno A, Bosetti M, Biasibetti A et al (2000) Prevention of pin track infection in external fixation with silver coated pins: clinical and microbiological results. J Biomed Mater Res 53:600-604

7. Stavlas P, Polyzois D (2005) Septic arthritis of the major joints of the lower limb after periarticular external fixation application: are conventional safe corridors enough to prevent it? Injury 36:239-247

8. Mahan J, Selgison D, Henry SL et al (1991) Factors in pin tract infections. Orthopaedics 14:305-308

9. Termaat MF, Raijmakers PG, Scholten HJ et al (2005) The accuracy of diagnostic imaging for the assessment of chronic osteomyelitis: a systematic review and meta-analysis. J Bone Joint Surg Am 87:2464-2471

10. Hughes DK (2003) Nuclear medicine and infection detection: the relative effectiveness of imaging with 111 In-Oxine-, 99mTcHMPAO-, and 99mTc-stannous fluoride colloid-labeled leukocytes and with 67 Ga-citrate. J Nucl Med Technol 31:196-201 